\title{
Motional spin relaxation in large electric fields
}

\author{
Riccardo Schmid, B. Plaster, and B. W. Filippone \\ California Institute of Technology, Pasadena, California 91125, USA
}

(Received 16 May 2008; published 1 August 2008)

\begin{abstract}
We discuss the precession of spin-polarized ultracold neutrons (UCNs) and ${ }^{3} \mathrm{He}$ atoms in uniform and static magnetic and electric fields and calculate the spin relaxation effects from motional $\mathbf{v} \times \mathbf{E}$ magnetic fields. Particle motion in an electric field creates a motional $\mathbf{v} \times \mathbf{E}$ magnetic field, which when combined with collisions produces variations of the total magnetic field and results in spin relaxation of neutron and ${ }^{3} \mathrm{He}$ samples. The spin relaxation times $T_{1}$ (longitudinal) and $T_{2}$ (transverse) of spin-polarized UCNs and ${ }^{3} \mathrm{He}$ atoms are important considerations in a new search for the neutron electric dipole moment at the Spallation Neutron Source $n \mathrm{EDM}$ experiment. We use a Monte Carlo approach to simulate the relaxation of spins due to the motional $\mathbf{v} \times \mathbf{E}$ field for UCNs and for ${ }^{3} \mathrm{He}$ atoms at temperatures below $600 \mathrm{mK}$. We find the relaxation times for the neutron due to the $\mathbf{v} \times \mathbf{E}$ effect to be long compared to the neutron lifetime, while the ${ }^{3} \mathrm{He}$ relaxation times may be important for the $n \mathrm{EDM}$ experiment.
\end{abstract}

DOI: 10.1103/PhysRevA.78.023401

PACS number(s): 33.25. $+\mathrm{k}, 76.60 .-\mathrm{k}, 82.56 . \mathrm{Na}, 51.20 .+\mathrm{d}$

\section{INTRODUCTION}

The phenomenon of longitudinal and transverse spin relaxation due to magnetic field inhomogeneities has been discussed extensively [1-5]. There, the fluctuating magnetic fields experienced by the particle spins during transport through magnetic field gradients coupled to collisions with other particles or a holding cell's walls lead to relaxation. For a combined magnetic and electric field configuration, another possible source of spin relaxation has been considered by Lamoreaux [6] and results from fluctuations in the motional magnetic field in the particle's rest frame. Here we present a more detailed discussion of this effect.

Transformation into the rest frame of a particle moving in an electric field gives rise to a motional magnetic field

$$
\boldsymbol{B}_{\boldsymbol{v}}=\mathbf{E} \times \mathbf{v} / c^{2} .
$$

The motional magnetic field is always perpendicular to both the direction of motion and the electric field. For parallel electric and magnetic field configurations (as are typically employed in particle electric dipole moment searches), the superposition of $\boldsymbol{B}_{\boldsymbol{v}}$ and a magnetic holding field $\boldsymbol{B}_{\mathbf{0}}$ leads to a small tilt in the total magnetic field $\boldsymbol{B}=\boldsymbol{B}_{0}+\boldsymbol{B}_{\boldsymbol{v}}$ (see Fig. 1), as the motional magnetic fields are typically very small relative to $B_{0}$. For example, in an upcoming search $(n \mathrm{EDM})$ [7] for the neutron electric dipole moment at the Spallation Neutron Source in the Oak Ridge National Laboratory, the motional magnetic field experienced by the neutrons from the $50 \mathrm{kV} / \mathrm{cm}$ electric field will be on the order of 5.6 $\times 10^{-4} \mathrm{mG}$ per $\mathrm{m} / \mathrm{s}$ of velocity, whereas the magnetic holding field $B_{0}$ will be about $10 \mathrm{mG}$.

Nevertheless, collisions result in an abrupt change in the velocity vector, causing the motional magnetic field $\boldsymbol{B}_{\boldsymbol{v}}$ to change in both direction and magnitude. Although the changes in orientation of the total magnetic field following a single collision are rather small (as $B_{v} \ll B_{0}$ ), after each collision, a particle's spin will precess about a slightly reoriented total magnetic field. After a large number of such collisions, each of these small "kicks" in the field will contribute to the overall global relaxation of a spin ensemble.
In the remainder of this paper, we calculate the magnitude of spin relaxation due to the $\mathbf{v} \times \mathbf{E}$ effect, especially as relevant for the conditions of the upcoming $n \mathrm{EDM}$ search, which will utilize ultracold neutrons (UCNs, neutrons with speeds $<7 \mathrm{~m} / \mathrm{s}$ ) and an in situ polarized ${ }^{3} \mathrm{He}$ "comagnetometer" immersed in a superfluid ${ }^{4} \mathrm{He}$ bath at temperatures in the range $100-600 \mathrm{mK}$. Spin relaxation times significantly longer than the neutron lifetime (limiting the measurement time) are desired.

The results in this paper are complementary to studies on spin relaxation due to magnetic field gradients [1-5] and calculations of the linear electric field geometric phase effects [8-10], both of which can be important considerations for the measurement of EDMs.

\section{THEORY}

\section{A. ${ }^{3} \mathrm{He}$ atoms}

We have followed the formalism of McGregor [5] to describe the relaxation process of the $\mathbf{v} \times \mathbf{E}$ motional field. This uses the field autocorrelation function to calculate longitudinal $\left(T_{1}\right)$ and transverse $\left(T_{2}\right)$ spin relaxation times. The spin relaxation times for particle spins due to a changing $B$ field can be calculated using the field autocorrelation function. With this method [5], the longitudinal spin relaxation rate can be expressed as

$$
\frac{1}{T_{1}}=\frac{\gamma^{2}}{2}\left[S_{B x}\left(\omega_{0}\right)+S_{B y}\left(\omega_{0}\right)\right],
$$

where $\gamma$ is the gyromagnetic ratio and $S_{B x}\left(\omega_{0}\right)$ and $S_{B y}\left(\omega_{0}\right)$ are the Fourier transforms of the field autocorrelation function. For a perturbing field $\widetilde{\boldsymbol{B}}$, the frequency spectrum is given by

$$
S_{B_{i}}(\omega)=\int_{-\infty}^{\infty}\left\langle\widetilde{B}_{i}(t) \widetilde{B}_{i}(t+\tau)\right\rangle e^{-\imath \omega \tau} d \tau
$$

for each of the fluctuating field components. The average inside the integral in Eq. (3) is calculated for particles in the 

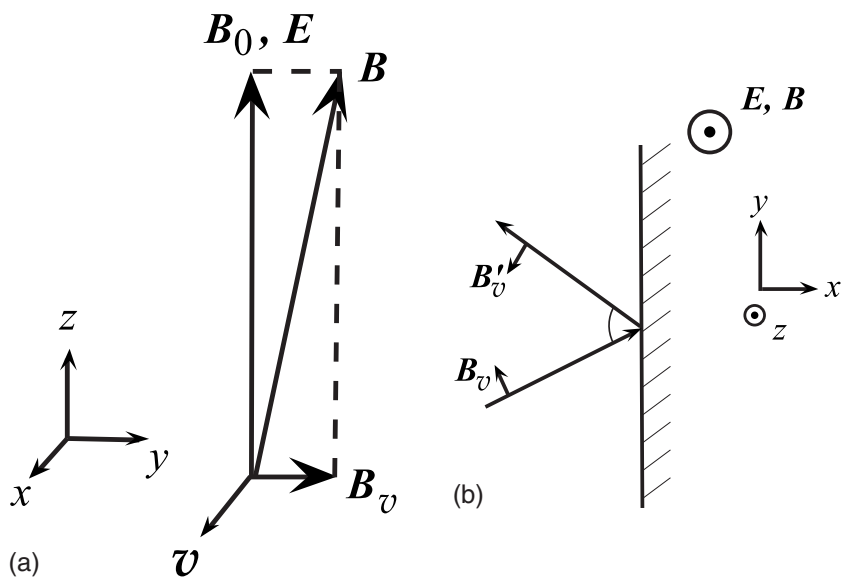

FIG. 1. (a) Total magnetic field $B$ as the superposition of the holding field $B_{0}$ and the $\mathbf{v} \times \mathbf{E}$ motional field $B_{v}$. (b) Rotation of motional field $B_{v}$ due to wall collision.

measurement cell colliding with the walls of the cell and with excitations in the superfluid ${ }^{4} \mathrm{He}$.

For the case of particles in a uniform magnetic holding field in the $\hat{z}$ direction and moving at velocity $\boldsymbol{v}$ in a uniform electric field $\boldsymbol{E}$ parallel to $\boldsymbol{B}_{0}$, the nonzero perturbation magnetic field terms are

$$
\begin{gathered}
\widetilde{B}_{x}=-E v_{y} / c^{2}, \\
\widetilde{B}_{y}=E v_{x} / c^{2} .
\end{gathered}
$$

The field autocorrelation function, for this case, is proportional to the velocity autocorrelation function as

$$
\begin{aligned}
& \left\langle\widetilde{B}_{x}(t) \tilde{B}_{x}(t+\tau)\right\rangle=\left\langle v_{y}(t) v_{y}(t+\tau)\right\rangle E^{2} / c^{4}, \\
& \left\langle\widetilde{B}_{y}(t) \widetilde{B}_{y}(t+\tau)\right\rangle=\left\langle v_{x}(t) v_{x}(t+\tau)\right\rangle E^{2} / c^{4} .
\end{aligned}
$$

For this work we adopt the velocity autocorrelation function [5]

$$
\left\langle v_{x}(t) v_{x}(t+\tau)\right\rangle=\left\langle v_{x}^{2}\right\rangle e^{-\tau / \tau_{c}},
$$

where $\tau_{c}$ is the mean time between collisions. Equation (6) is applicable in determining the frequency spectrum when the Larmor frequency $\omega_{0}$ is larger than $1 / \tau_{D}$, where $\tau_{D}$ is the diffusion time. This is the case for ${ }^{3} \mathrm{He}$ atoms at temperatures $T>400 \mathrm{mK}$, where, as discussed in Sec. III A, collisions with excitations in the superfluid are frequent and atoms take a relatively long time to travel to the walls of the holding cell. The corresponding field autocorrelation frequency spectrum is

$$
S_{B x}(\omega)=\frac{2\left\langle v_{y}^{2}\right\rangle \tau_{c}}{1+\omega^{2} \tau_{c}^{2}} \frac{E^{2}}{c^{4}},
$$

and a similar formula for $S_{B y}(\omega)$. With Eq. (2), we find the longitudinal spin relaxation time to be

$$
T_{1}=\frac{3}{2} \frac{1}{\gamma^{2}} \frac{c^{4}}{E^{2} v_{\mathrm{rms}}^{2} \tau_{c}}\left(1+\omega_{0}^{2} \tau_{c}^{2}\right),
$$

where we have used $\left\langle v_{x}^{2}\right\rangle+\left\langle v_{y}^{2}\right\rangle=2 v_{\mathrm{rms}}^{2} / 3$. As discussed in Sec. III A, the mean time between collisions drops dramatically as the temperature of the ${ }^{3} \mathrm{He}$ sample increases, and, in the $n \mathrm{EDM}$ setting, $\omega_{0} \tau_{c} \ll 1$ for $T>300 \mathrm{mK}$, so that Eq. (8) can be approximated to be

$$
T_{1} \simeq \frac{3}{2} \frac{1}{\gamma^{2}} \frac{c^{4}}{E^{2} v_{\mathrm{rms}}^{2} \tau_{c}},
$$

leaving the relaxation times independent of the magnetic holding field $B_{0}$ for this regime.

The transverse spin relaxation rate can be expressed as [5]

$$
\frac{1}{T_{2}}=\frac{1}{2 T_{1}}+\frac{\gamma^{2}}{2} S_{B z}(0),
$$

but, for the case of $\mathbf{v} \times \mathbf{E}$ fields in which the perturbation field has no $z$ component, the term $S_{B z}(0)=0$, so we find that $T_{2}=2 T_{1}$.

\section{B. Ultracold neutrons}

Although Eq. (2) can be applied to any perturbative field, as long as the proper expression for the autocorrelation function is used, we note that the earlier formalism detailed in [1] is useful for the special case of UCNs whose velocities do not change in the $n \mathrm{EDM}$ measurement cells. Following their procedure to calculate the spin relaxation in inhomogeneous fields, which does not rely on the field autocorrelation function, the expression for the longitudinal relaxation time $T_{1}$ due to the motional $\mathbf{v} \times \mathbf{E}$ field can be calculated to be

$$
T_{1}=\frac{3}{4} \frac{(1.19)^{2} \lambda B_{0}^{2} c^{4}}{v^{3} E^{2}} \frac{1+\left(\omega_{0} \tau_{c}\right)^{2}}{\left(\omega_{0} \tau_{c}\right)^{2}} .
$$

This formula is equivalent to Eq. (8), apart from small constant terms. It is also interesting to note that, for the case of UCNs in the $n \mathrm{EDM}$ experiment, the mean free path $\lambda$ in Eq. (11) is a constant of the geometry of the measurement cell, since UCNs do not interact with other particles. In addition, $\omega_{0} \tau_{c}>1$ for UCN speeds, so that the last factor in Eq. (11) is roughly constant and of order unity. In this case, Eq. (11) can be approximated to be

$$
T_{1} \simeq \frac{3}{4} \frac{(1.19)^{2} \lambda B_{0}^{2} c^{4}}{v^{3} E^{2}},
$$

emphasizing the $T_{1} \propto 1 / v^{3}$ relation. The relaxation time can be lengthened by increasing the magnitude of the magnetic holding field $B_{0}$. However, as seen in Sec. III B, the motional spin relaxation times for UCNs in the $n \mathrm{EDM}$ experiment are long compared to the neutron lifetime.

\section{SIMULATION}

We have simulated the spin relaxation of UCNs and ${ }^{3} \mathrm{He}$ atoms due to the $\mathbf{v} \times \mathbf{E}$ effect for uniform and static magnetic and electric fields. Our studies are based on the precession of 
classical spins in a magnetic field. Neutrons (and ${ }^{3} \mathrm{He}$ atoms) are confined inside the rectangular $n \mathrm{EDM}$ measurement cell of dimensions $\Delta x=10.2 \mathrm{~cm}, \Delta y=50 \mathrm{~cm}$, and $\Delta z=7.6 \mathrm{~cm}$, where both magnetic and electric fields are oriented in the $\hat{z}$ direction. The mean distance between wall collisions can be approximated by $\lambda_{w} \simeq 4 V / A$ for a rectangular measurement cell, where $V$ and $A$ are the volume and the surface area of the cell, respectively. For the $n \mathrm{EDM}$ cell, $\lambda_{w} \simeq 8 \mathrm{~cm}$, which is comparable to the mean distance between wall collisions of $\lambda_{\text {sim }}=7.5 \mathrm{~cm}$ obtained from the simulations. As discussed in Sec. III A, ${ }^{3} \mathrm{He}$ atoms can interact with excitations in the superfluid, so that the mean time between collisions, $\tau_{c}$, is determined by both diffusion and wall collisions.

As we neglect the effect of gravity, changes in velocity are due only to collisions; hence, between collisions, the total magnetic field experienced in the rest frame of a particle stays constant. We have used an analytic solution to the equation of motion of the spin in a constant magnetic field,

$$
\dot{\boldsymbol{\sigma}}=\gamma \boldsymbol{\sigma} \times \boldsymbol{B},
$$

to calculate the spin precession between collisions. The uniform magnetic field solution to Eq. (13) can be found to be

$$
\begin{aligned}
\boldsymbol{\sigma}(t)= & \left(\boldsymbol{\sigma}_{0}-\frac{\left(\boldsymbol{\sigma}_{0} \cdot \boldsymbol{B}\right) \boldsymbol{B}}{B^{2}}\right) \cos (\omega t) \\
& +\frac{\boldsymbol{\sigma}_{0} \times \boldsymbol{B}}{B} \sin (\omega t)+\frac{\left(\boldsymbol{\sigma}_{0} \cdot \boldsymbol{B}\right) \boldsymbol{B}}{B^{2}} .
\end{aligned}
$$

We have studied the case of totally diffuse wall collisions for both UCNs and ${ }^{3} \mathrm{He}$ atoms. The diffuse wall collisions are in part responsible for the spin relaxation. Our simulations have shown that, for the $\mathbf{v} \times \mathbf{E}$ studies described in this paper, purely specular wall collisions, in the absence of particle diffusion, do not result in significant longitudinal relaxation of the spin polarization. In this case, the velocity of a particle after a wall collision is highly correlated to its initial velocity and the times between wall collisions are repetitive, so that the motional field $B_{v}$ changes in a repeatable way and does not, generally, allow the spins to precess far from their initial states. We were able to determine from the simulation data that only a small fraction $\left(<10^{-3}\right)$ of particle spins were precessing away from their initial states for the case of specular wall collisions. We believe that this effect occurs because these particles are traveling in resonant paths, where the correlation of velocities and precession times between wall collisions is such as to maximally rotate the spins out of alignment. Furthermore, the walls of the measurement cells in the $n \mathrm{EDM}$ experiment are not likely to be purely specular.

In the simulations we track the spin state of each particle and calculate the longitudinal, $T_{1}$, and transverse, $T_{2}$, relaxation times due to the $\mathbf{v} \times \mathbf{E}$ effect for single speed UCNs and for ${ }^{3} \mathrm{He}$ atoms at temperatures below $600 \mathrm{mK}$. In the longitudinal spin relaxation simulations, particles start with their spins aligned with the holding field $B_{0}$. Due to the $\mathbf{v}$ $\times \mathbf{E}$ effect, the ensemble of spins relaxes exponentially with a time constant $T_{1}$, as shown in Fig. 2. In contrast, in the transverse spin relaxation simulations, particles start with their spins perpendicular to the holding field $B_{0}$ and the spins relax with an exponential time constant $T_{2}$.

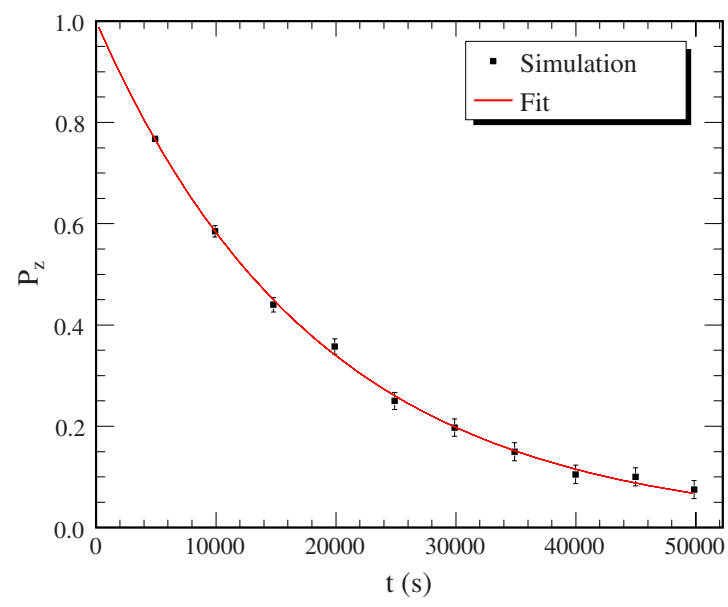

FIG. 2. (Color online) Simulation results for the longitudinal polarization $P_{z}$ for a ${ }^{3} \mathrm{He}$ sample in a thermal bath at $T=100 \mathrm{mK}$ vs time. An exponential fit to the simulation data is also shown. The fit yields the relaxation time $T_{1}$.

\section{A. ${ }^{3} \mathrm{He}$ atoms}

We have simulated the spin relaxation due to the $\mathbf{v} \times \mathbf{E}$ effect in a magnetic holding field $B_{0}=10 \mathrm{mG}$ and an electric field $E_{0}=50 \mathrm{kV} / \mathrm{cm}$, for a sample of ${ }^{3} \mathrm{He}$ atoms at temperatures below $600 \mathrm{mK}$. Unlike UCNs (see Sec. III B), whose speeds were constant in our simulations, the ${ }^{3} \mathrm{He}$ sample obeys a Maxwell-Boltzmann velocity distribution of the form

$$
f(v)=4 \pi\left(\frac{M_{\star}}{2 \pi k T}\right)^{3 / 2} v^{2} e^{-M_{\star} v^{2} / 2 k T} d v,
$$

where $T$ is the temperature of the sample, $k$ is the Boltzmann constant, and $M_{\star} \simeq 2.2 M_{3}$ is the effective mass of ${ }^{3} \mathrm{He}$ atoms in superfluid ${ }^{4} \mathrm{He}$, with $M_{3}$ the ${ }^{3} \mathrm{He}$ atomic mass [11]. The mean speed of ${ }^{3} \mathrm{He}$ atoms is $\bar{v}=\langle v\rangle=\sqrt{8 k T / \pi M_{\star}}$, while the root mean square speed is $v_{\text {rms }} \equiv \sqrt{\left\langle v^{2}\right\rangle}=\sqrt{3 k T / M_{\star}}$.

The collisions with the walls and interactions with the superfluid were implemented to be totally diffuse. In addition, we included thermalization of ${ }^{3} \mathrm{He}$ atoms, so that the magnitude of the velocity is randomly sampled from the original velocity distribution [Eq. (15)] after each collision with either phonons or the walls of the measurement cell.

The ${ }^{3} \mathrm{He}$ atoms can interact with excitations in the superfluid ${ }^{4} \mathrm{He}$ in the $n$ EDM cell. Due to the low fractional density of ${ }^{3} \mathrm{He}$ atoms, typically on the order of one ${ }^{3} \mathrm{He}$ atom per $10^{10}$ atoms of ${ }^{4} \mathrm{He}$, collisions with other ${ }^{3} \mathrm{He}$ atoms are negligible. The mass diffusion coefficient for ${ }^{3} \mathrm{He}$ atoms in superfluid He depends sensitively on the sample temperature and was found experimentally [12] to be

$$
D=\frac{(1.6 \pm 0.2) \mathrm{cm}^{2} \mathrm{~s}^{-1}}{[T(\mathrm{~K})]^{7}} .
$$

Since the phonon velocity in superfluid ${ }^{4} \mathrm{He}$ is much higher than the velocity of individual atoms [10], the mean time between phonon interactions with ${ }^{3} \mathrm{He}$ atoms in the 
TABLE I. Temperature dependence of velocity and mean free path for a sample of ${ }^{3} \mathrm{He}$ atoms at selected temperatures. The $4 \mathrm{mK}$ point represents ${ }^{3} \mathrm{He}$ velocities comparable to those of UCNs.

\begin{tabular}{lcccc}
\hline \hline$T(\mathrm{mK})$ & $\bar{v}(\mathrm{~m} / \mathrm{s})$ & $\lambda_{p}(\mathrm{~cm})$ & $\omega_{0} \tau_{D}$ & $\omega_{0} \tau_{c}$ \\
\hline 4 & 3.573 & $\gg 10^{3}$ & 4.45 & 4.45 \\
50 & 12.63 & $\gg 10^{3}$ & 1.26 & 1.26 \\
100 & 17.86 & $\gg 10^{3}$ & 0.890 & 0.890 \\
200 & 25.26 & 126 & 0.668 & 0.593 \\
300 & 30.94 & 6.02 & 1.18 & 0.224 \\
400 & 35.73 & 0.696 & 5.43 & $3.65 \times 10^{-2}$ \\
500 & 39.94 & 0.131 & 24.2 & $6.55 \times 10^{-3}$ \\
600 & 43.76 & $3.33 \times 10^{-2}$ & 85.5 & $1.54 \times 10^{-3}$ \\
\hline \hline
\end{tabular}

measurement cell is constant for a given temperature and independent of the instantaneous ${ }^{3} \mathrm{He}$ velocity and can be expressed as [10]

$$
\tau_{p}=\frac{3 D}{v_{\mathrm{rms}}^{2}} .
$$

The effective mean time between collisions, $\tau_{c}$, which takes into account both superfluid excitations and wall collisions, is

$$
\frac{1}{\tau_{c}}=\frac{\bar{v}}{\lambda_{w}}+\frac{1}{\tau_{p}} .
$$

The mean time $\tau_{D}$ that it takes for ${ }^{3} \mathrm{He}$ atoms to diffuse to the walls of the measurement cell is useful for defining the regime in which the formalism described by Eq. (6) is valid, and can be expressed as

$$
\tau_{D}=\frac{R^{2}}{\tau_{c} v_{\mathrm{rms}}^{2}},
$$

where $R \simeq 8 \mathrm{~cm}$ is the characteristic size of the measurement cell, or the radius for a spherical cell.

Table I shows the mean distance between interactions with excitations in the superfluid $\lambda_{p}=\tau_{p} \bar{v}$, for selected simulation temperatures, along with other relevant quantities. In the low temperature regime $(T<200 \mathrm{mK})$ the mean distance between collisions with excitations $\lambda_{p}$ is longer than the dimensions of the $n \mathrm{EDM}$ measurement cell, so wall collisions dominate and the mean free path is the mean distance between wall collisions. In the high-temperature regime $(T>300 \mathrm{mK}) \quad \lambda_{p} \lesssim 5 \mathrm{~cm}$, so collisions with excitations dominate. When the collision frequency is high relative to the precession frequency, the spin relaxation times due to the $\mathbf{v} \times \mathbf{E}$ effect increase. In the mid-temperature regime $(T \sim 100-300 \mathrm{mK})$, where the quantity $\omega_{0} \tau_{D}$ is of order unity, Eq. (7) is not a good approximation for the frequency spectrum, and this is where we see the largest deviation between the simulations and the predicted relaxation times. For $\omega_{0} \tau_{D} \ll 1$, a full diffusion theory calculation is necessary to determine the frequency spectrum. In any case, the $n \mathrm{EDM}$

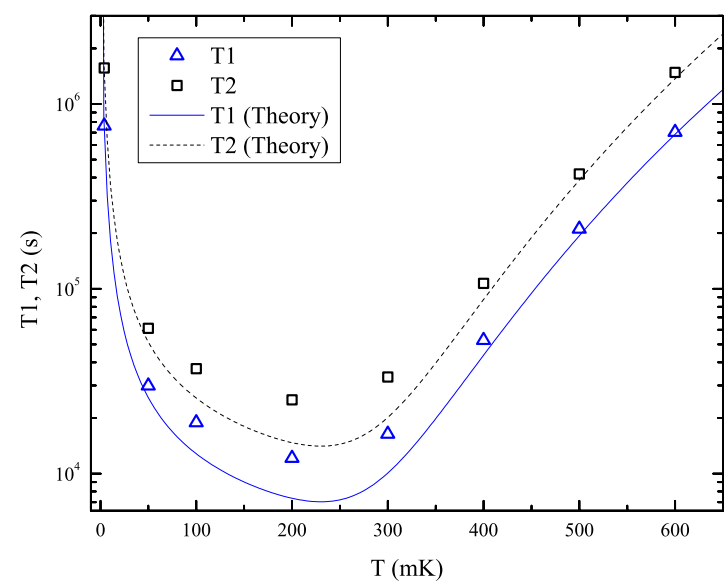

FIG. 3. (Color online) Spin relaxation times $T_{1}$ and $T_{2}$ for ${ }^{3} \mathrm{He}$ atoms as a function of temperature, plotted together with the theoretical formulas derived from Eq. (8).

geometry and field configuration is such that the quantity $\omega_{0} \tau_{D} \gtrsim 1$ for all ranges of temperatures in our study, keeping the deviation small.

We analyzed the data from our simulations to calculate the spin relaxation times $T_{1}$ and $T_{2}$ for ${ }^{3} \mathrm{He}$ atoms for temperatures below $600 \mathrm{mK}$. The results are shown in Fig. 3, where relaxation times are plotted as a function of temperature. The simulation data agree with the theoretical diffusion calculations, especially for temperatures higher than $400 \mathrm{mK}$, in which the collision frequency $1 / \tau_{c}$ is large and the diffusion times $\tau_{D}$ are long. The simulation data also agree with the relation $T_{2}=2 T_{1}$.

Our calculations and simulations have shown that the longitudinal spin relaxation times $T_{1}$ for ${ }^{3} \mathrm{He}$ atoms at temperatures in the range $100-400 \mathrm{mK}$ can be as short as $10^{4} \mathrm{~s}$. These relaxation times are comparable to the measurement time of $n \mathrm{EDM}$ and may be an important component of the total relaxation rate. In this regard, the temperature is a parameter of the measurement that can be chosen to optimize spin relaxation times.

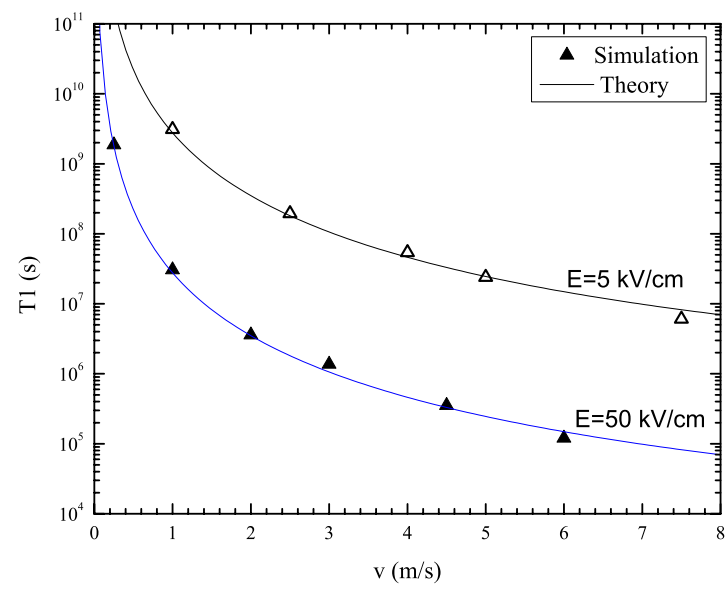

FIG. 4. (Color online) Simulation results for longitudinal spin relaxation times $T_{1}$ for UCNs as a function of velocity and with different electric field strengths. The simulation data agree with the theoretical formalism described by Eq. (11). 


\section{B. Ultracold neutrons}

We have simulated the spin relaxation due to the $\mathbf{v} \times \mathbf{E}$ effect for UCNs for the conditions in the $n \mathrm{EDM}$ experiment. The measurement cell, as designed for the $n$ EDM experiment, has a UCN Fermi potential of $134 \mathrm{neV}$, which is equivalent to a maximum UCN velocity of $3.6 \mathrm{~m} / \mathrm{s}$ and constitutes an upper limit for the velocity spectrum of UCNs.

The relaxation times are found to be shorter for higher UCN speeds, as discussed in Sec. II B. We have simulated UCNs at different velocities (see Fig. 4), experiencing diffuse collisions with the walls of the cell. The UCNs do not interact with other particles in the cell so their speed stays constant in our simulations. The $n$ EDM magnetic field configuration was used, with $B_{0}=10 \mathrm{mG}$.

The results from the UCN simulations agree with the theoretical results $[\mathrm{Eq} .(11)]$ in the regime in which collision frequencies are low with respect to the Larmor frequency. The expected $1 / v^{3}$ dependence of $T_{1}$ for UCNs can be seen in Fig. 4, for two values of $E=5$ and $50 \mathrm{kV} \mathrm{cm}^{-1}$. The relevant relaxation times for UCNs with speeds below $4 \mathrm{~m} / \mathrm{s}$ are on the order of $10^{5} \mathrm{~s}$, for the configuration of the $n \mathrm{EDM}$ measurement. These relaxation times are much longer than the neutron lifetime and should not constitute a limiting factor for the experiment.

\section{CONCLUSION}

We have studied the longitudinal and transverse spin relaxation times $T_{1}$ and $T_{2}$ from motional $\mathbf{v} \times \mathbf{E}$ fields. The results from Monte Carlo simulations and theoretical calculations of the relaxation times are shown to be in agreement. We investigated this phenomenon within the context of the operating parameters for an upcoming search for the neutron electric dipole moment utilizing ultracold neutrons and a polarized ${ }^{3} \mathrm{He}$ comagnetometer. For UCNs, the relaxation times were found to be on the order of $10^{6} \mathrm{~s}$, much longer than the neutron lifetime. In contrast, our calculations have shown that the relaxation times for ${ }^{3} \mathrm{He}$ atoms will vary strongly with temperature, and can be as low as $10^{4} \mathrm{~s}$. Thus, consideration of the relaxation effects from $\mathbf{v} \times \mathbf{E}$ motional magnetic fields can influence the choice of the experiment's operating temperature.

\section{ACKNOWLEDGMENTS}

We would like to thank M. Hayden for helpful discussions. The work in this paper was supported in part by the U.S. National Science Foundation, Grant No. PHY-0555674.
[1] R. L. Gamblin and T. R. Carver, Phys. Rev. 138, A946 (1965).

[2] L. D. Schearer and G. K. Walters, Phys. Rev. 139, A1398 (1965).

[3] G. D. Cates, S. R. Schaefer, and W. Happer, Phys. Rev. A 37, 2877 (1988).

[4] G. D. Cates, D. J. White, T.-R. Chien, S. R. Schaefer, and W. Happer, Phys. Rev. A 38, 5092 (1988).

[5] D. D. McGregor, Phys. Rev. A 41, 2631 (1990).

[6] S. K. Lamoreaux, Phys. Rev. A 53, R3705 (1996).
[7] SNS Neutron EDM Experiment $n$ EDM, URL http:// p25ext.lanl.gov/edm/edm.html

[8] J. M. Pendlebury et al., Phys. Rev. A 70, 032102 (2004).

[9] S. K. Lamoreaux and R. Golub, Phys. Rev. A 71, 032104 (2005).

[10] A. L. Barabanov, R. Golub, and S. K. Lamoreaux, Phys. Rev. A 74, 052115 (2006).

[11] E. Krotscheck, M. Saarela, K. Schörkhuber, and R. Zillich, Phys. Rev. Lett. 80, 4709 (1998).

[12] S. K. Lamoreaux et al., Europhys. Lett. 58, 718 (2002). 